\title{
Relación de mating intelligence con personalidad, satisfacción vital, orientación socio-sexual y estrategias de historia de vida en una muestra de adultos de Asunción
}

\author{
Iliana Croskey ${ }^{1}$, Jonathan Ayala ${ }^{1}$
}

\begin{abstract}
Resumen
Introducción: Ésta investigación fue parte del proyecto 14-INV-380 PROCIENCIA/ CONACYT. "El poder predictivo combinado de los factores y las facetas de personalidad, inteligencia fluida y situación laboral con respecto al rendimiento académico". Se enfocó en determinar las asociaciones entre el constructo mating intelligence, modelo teórico propuesto por Geher, Camargo, y O’Rourke (2008) que comprende un conjunto de habilidades cognitivas diseñadas para la reproducción sexual, con los rasgos de personalidad (conjuntos de rasgos y cualidades que integran a una persona), satisfacción vital (sensación subjetiva de bienestar físico, psicológico y social), orientación socio-sexual (predisposición hacia las tendencias sexuales sin compromiso) y estrategias de historia de vida (patrones de conductas adaptados para la supervivencia y reproducción). La enmarcación teórica se basó en las teorías de la biología, psicología evolutiva, psicología de la personalidad y psicología positiva. El estudio pretendió replicar y ampliar el trabajo original de Geher, G. y Kaufman, S., (2011) con título Predicting Preferences for Sex Acts: Which Traits Matter Most, and Why?, para lo cual se utilizó a la población paraguaya. Asimismo el estudio consistió en analizar las correlaciones entre Mating intelligence con los rasgos de Personalidad, Satisfacción vital, Orientación socio-sexual y estrategias de historia de vida en una muestra de adultos de 18 a 56 años de edad del Paraguay. Acerca de los antecedentes, se tuvo en cuenta que dentro del territorio nacional son escasos los estudios acerca de factores de personalidad y conductas de cortejo.
\end{abstract}

Objetivo: Determinar la relación de mating intelligence con personalidad, satisfacción vital, orientación socio-sexual, estrategias de historia de vida y conductas de cortejo en una muestra de adultos de Asunción.

Material y Método: Durante la recolección de datos se utilizó la Mating intelligences scale, diseñado para brindar una guía aproximada acerca de cuán

1. Universidad del Cono Sur de las Américas y Universidad. Psicología/Humanidades.

Círculo de Investigación en Psicología de la Personalidad y Diferencias Individuales

Trabajo de tesis presentado el 20 de junio del 2018 en la Universidad del Cono Sur de las Américas.

E-mail: ilicroskey@hotmail.com

DOI: 10.26885/rcei.foro.2018.114 
efectiva es una persona en sus relaciones interpersonales, el test IPIP Big Five, el inventario de Orientación sociosexual Revisado (SOI-R), el inventario K-SF42 , la versión reducida, la escala de satisfacción con la vida (SWLS) y la escala de cortejo. El alcance del estudio fue descriptivo y correlacional, de diseño no experimental el cual contó con una muestra total de 251 personas, que fueron seleccionadas mediante el muestreo no probabilístico por conveniencia.

Resultados: El principal resultado del estudio es la asociación significativa entre inteligencia de cortejo con los factores responsabilidad, neuroticismo, extraversión y apertura a la experiencia; asimismo, con satisfacción vital, orientación socio-sexual y estrategias de historias de vida.

Conclusión: Se concluye que existen relaciones entre mating intelligence, personalidad, satisfacción vital, orientación socio-sexual, estrategias de historia de vida y las preferencias sexuales.

Palabras clave: mating intelligence, personalidad, orientación socio-sexual.

\section{Referencias}

Gangestad, S. W., y Simpson, J.A. (2000). The evolution of human mating: The role of trade-offs and strategic pluralism. Behavioral and Brain Sciences, 23, 675-687.

Geher, G. y Kaufman, S. (2011). Predicting Preferences for Sex Acts: Which Traits Matter Most, and Why? Ep Journal, 9(03), 371-389

Geher, G., Camargo, M. y O'Rourke, S. (2008). Mating Intelligence: an integrative model and future research directions. En Geher G. y Miller G. (Eds.), Mating intelligence: sex, relationships and the mind's reproductive system (pp.395-424). New York, Estados Unidos: Lawrence Erlbaum Associate.

O’Brien, D., Geher, G., Gallup, A., García, J. y Kaufman, S. (2010). Self-perceveived mating intelligence predicts sexual behavior in college students: empirical validation of a theoretical construct. Imagination, cognition and personality, 29(04), 341-362.

Penke, L. (2008). The revised Sociosexual Orientation Inventory. Journal of Personality and Social Psychology, 95, 1113-1135. 